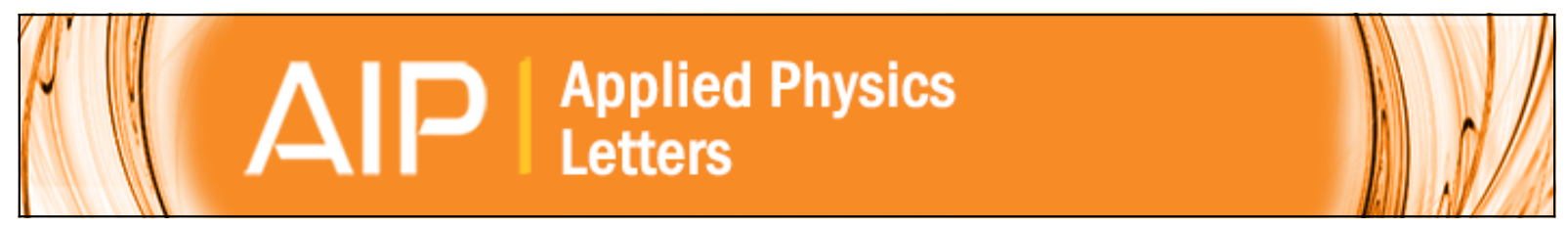

\title{
Irreversible extinction of ferroelectric polarization in P(VDF-TrFE) thin films upon melting and recrystallization
}

Youn Jung Park, Seok Ju Kang, Cheolmin Park, Kap Jin Kim, Han Sup Lee, Moon Sook Lee, U-In Chung, and In Jun Park

Citation: Applied Physics Letters 88, 242908 (2006); doi: 10.1063/1.2207831

View online: http://dx.doi.org/10.1063/1.2207831

View Table of Contents: http://scitation.aip.org/content/aip/journal/apl/88/24?ver=pdfcov

Published by the AIP Publishing

\section{Articles you may be interested in}

In situ observation of the nanocrystal growth and their piezoelectric performance change in P(VDF-TrFE) films by hot stage piezoresponse force microscopy

J. Appl. Phys. 113, 187210 (2013); 10.1063/1.4801971

Tuned dielectric, pyroelectric and piezoelectric properties of ferroelectric $\mathrm{P}(\mathrm{VDF}-\mathrm{TrFE})$ thin films by using mechanical loads

J. Appl. Phys. 111, 044102 (2012); 10.1063/1.3685750

Ferroelectric switching dynamics in VDF-TrFE copolymer thin films spin coated on Si substrate

J. Appl. Phys. 105, 061636 (2009); 10.1063/1.3055411

Crystalline structure and transition behavior of recrystallized-irradiated P(VDF-TrFE) 65/35 copolymer

J. Appl. Phys. 97, 014102 (2005); 10.1063/1.1827918

Dependence of threshold thickness of crystallization and film morphology on film processing conditions in poly(vinylidene fluoride-trifluoroethylene) copolymer thin films

J. Appl. Phys. 92, 3111 (2002); 10.1063/1.1503395

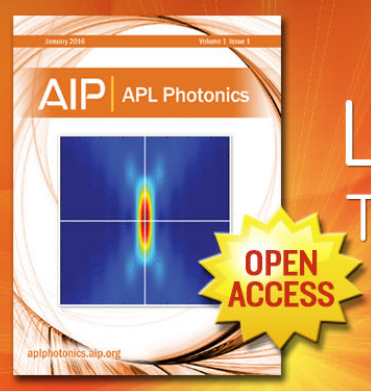

Launching in 2016! The future of applied photonics research is here 


\title{
Irreversible extinction of ferroelectric polarization in P(VDF-TrFE) thin films upon melting and recrystallization
}

\author{
Youn Jung Park, Seok Ju Kang, and Cheolmin Park ${ }^{\mathrm{a})}$ \\ Department of Materials Science and Engineering, Yonsei University, Seoul 120-749, Korea \\ Kap Jin Kim \\ College of Environment and Applied Chemistry, Kyung Hee University, Gyeonggi-do 446-701, Korea \\ Han Sup Lee \\ Department of Textile Engineering, Inha University, Incheon 402-751, Korea \\ Moon Sook Lee and U-In Chung \\ New Memory Process Development Team, Samsung Electronics Giheung Factory, Yongin-si, \\ Gyeonggido 446-711, Korea \\ In Jun Park \\ Interface Materials and Engineering Laboratory, Korea Research Institute of Chemical Technology, \\ Jang-dong 100, Yuseong, Daejeon 305-343, Korea
}

(Received 3 December 2005; accepted 13 April 2006; published online 16 June 2006)

\begin{abstract}
We observed the irreversible extinction of ferroelectric polarization in spun coated poly(vinylidene fluoride-co-trifluoroethylene) thin films upon melting and recrystallization. We investigate the alteration of the ferroelectric properties correlated with the preferred polymer crystal orientation with respect to the electrodes using grazing incident scattering, spectroscopy, and electron microscopes. Heat treatment above melting point gave rise to the significant reduction of the ferroelectric performance mainly caused by the modification of molecular orientation of polymer crystals whose $c$ and $b$ axes are perpendicular and parallel to the electrode surface, respectively, leading to almost zero effective electric field. () 2006 American Institute of Physics.
\end{abstract}

[DOI: $10.1063 / 1.2207831]$

With the tremendous demand for an electrically addressable memory technology that can be placed into organic integrated circuits, ${ }^{1}$ ferroelectric poly(vinylidene fluoride-cotrifluoroethylene) $\mathrm{P}(\mathrm{VDF}-\mathrm{TrFE})$ copolymers were often introduced to have possibilities of application for the nonvolatile and low cost memory device. ${ }^{2,3}$ A number of studies ${ }^{4-9}$ have been focused on the ferroelectric behavior: permanent polarization, ${ }^{4,5}$ switching time, ${ }^{6}$ coercive voltage, ${ }^{7}$ crystallinity in ultrathin films, ${ }^{8}$ interfacial layer control of ferroelectric capacitors, ${ }^{9}$ and electroactive polymers irradiated by electron beam. ${ }^{10,11}$ For both low voltage switching below $5 \mathrm{~V}$ and low fabrication cost, the polymer should be solution processed with the film thickness of less than $100 \mathrm{~nm}$ due to its high coercive field $\left(E_{c}\right)$ of approximately $50 \mathrm{MV} / \mathrm{m}$. The efforts to reduce the film thickness for the device operating at low voltage encounter the difficulties that there exists the threshold film thickness below which polymer capacitors have dramatically low crystallinity and consequently increase the switching time. ${ }^{12}$ For overcoming these problems, many researchers have concentrated on the issues of increasing crystallinity ${ }^{13}$ and/or controlling crystal orientation. ${ }^{14}$ Thermal treatment including the process of consecutive annealing has been considered to be one of the most effective processes so as to enhance the ferroelectric properties. ${ }^{15}$ However, most works have been focused on the effect of annealing temperature in the range of Curie $\left(T_{C}\right)$ and melting temperature $\left(T_{m}\right)$. In order to adopt the polymer ferroelectrics into the current integrated process which requires high tem-

\footnotetext{
${ }^{\text {a) }}$ Author to whom correspondence should be addressed; electronic mail:
} cmpark@yonsei.ac.kr perature well above $T_{m}$, the ferroelectric properties become more important, depending on thermal history from melt recrystallization.

Here we present the effect of thermal treatment of thin $\mathrm{P}(\mathrm{VDF}-\mathrm{TrFE})$ films on ferroelectric properties. The improved remnant polarization observed in the samples annealed at temperatures between $T_{C}$ and $T_{m}$ completely disappears when the samples are heated above $T_{m}$ and recrystallized through various pathways. Extinction of the remnant polarization of the melt-recrystallized samples arises from the modification of the molecular chain orientation upon recrystallization presumably due to the preferential crystal face interaction to the electrode surface.

We employed a P(VDF-TrFE) copolymer with $27.5 \%$ TrFE which was kindly supplied from MSI Sensor, PA, USA. The crystallinity index calculated from the experimental melting enthalpy and the equilibrium enthalpy of a perfect crystal of PVDF $(104.7 \mathrm{~J} / \mathrm{g})$ was nearly $60 \% . T_{m}$ and $T_{C}$ of $\mathrm{P}(\mathrm{VDF}-\mathrm{TrFE})$ were 150 and $80{ }^{\circ} \mathrm{C}$, respectively. Thin film formation was done by spin coating with 1\%-3\% P(VDFTrFE) solution in methyl ethyl ketone (MEK). Thickness of the fabricated film was measured by ellipsometry (Gaertner Scientific Co. USA). Heat treatment for the films was performed on the heating stage (Linkam 600, UK) with different steps demonstrated in Fig. 1(a). Metal/P(VDF-TrFE)/metal capacitors were made with highly doped silicon (100) substrate as the bottom electrode. Aluminum top electrodes were evaporated on polymer films using a shadow mask with holes of $200 \mu \mathrm{m}$ in diameter under a pressure of $\sim 10^{-6} \mathrm{mbar}$ and a rate of $\sim 0.1 \mathrm{~nm} / \mathrm{s}$. Ferroelectric properties were obtained using a RT66A unit (Radiant Technolo- 
(a)

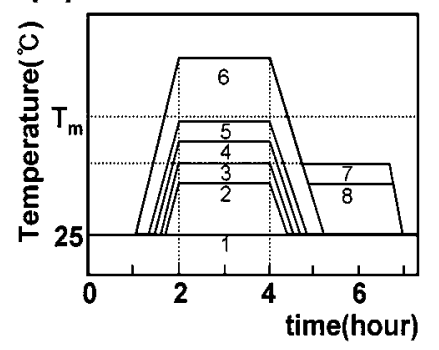

(c)

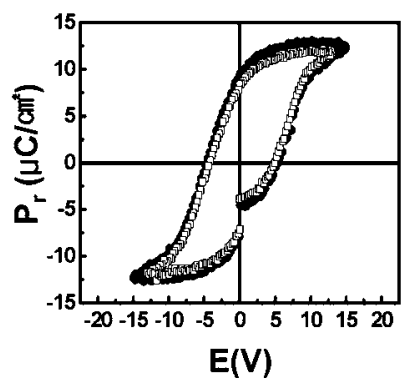

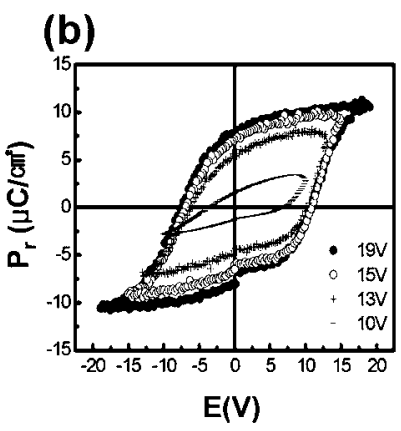

(d)

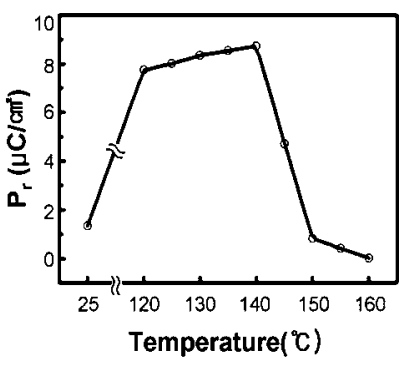

FIG. 1. (Color online) (a) Various routes for annealing treatments as a function of time. Each film was experienced thermal annealing treatment at different temperatures via the routes of 1-8. (b) Polarization $P$ vs applied voltage $E$ hysteresis loops of a P(VDF-TrFE) polymer capacitor. (c) Ferroelectric hysteresis loops of a $70 \mathrm{~nm}$ thin $\mathrm{P}(\mathrm{VDF}-\mathrm{TrFE})$ polymer film. (d) The remnant polarization $\left(P_{r}\right)$ as a function of annealing temperature.

gies). The structures of the films were characterized by scanning electron microscope (SEM, HITACHI S-2700), transmission electron microscope (TEM, Phillips X60), and the grazing-incidence $\mathrm{x}$-ray diffraction (GIXD). Infrared grazing-incidence reflection absorption spectra (GIRAS) were collected using Bruker-IFS66V spectrometer.

Figure 1(b) shows a typical hysteresis loop of $200 \mathrm{~nm}$ $\mathrm{P}(\mathrm{VDF}-\mathrm{TrFE})$ film spun coated from $3 \mathrm{wt} \%$ solution and annealed at $120^{\circ} \mathrm{C}$ for $2 \mathrm{~h}$. The remnant polarization $\left(P_{r}\right)$ and coercive voltage $\left(V_{c}\right)$ in a saturated curve are approximately $7.8 \mu \mathrm{C} / \mathrm{cm}^{2}$ and $11 \mathrm{~V}$ corresponding to coercive electric field $\left(E_{c}\right)$ of approximately $50 \mathrm{MV} / \mathrm{m}$, when the applied voltage is above $\pm 15 \mathrm{~V}$, which is consistent with that reported by others. ${ }^{4,9}$ The very low switching voltage of $4.9 \mathrm{~V}$ was detected in the $70 \mathrm{~nm}$ film [Fig. 1(c)]. Further lowering the thickness caused the significant reduction of the polarization due to the reduced crystallinity.

The polarization increased slightly with the annealing temperature before crystal melting, as shown in Fig. 1(d). Heat treatment of the samples above $T_{m}$ dramatically reduced the remnant polarization irrespective of the pathways of recrystallization: one rapidly cooled to room temperature (sample 6), others slowly cooled with the rate of $2{ }^{\circ} \mathrm{C} / \mathrm{min}$, to 120 and $130{ }^{\circ} \mathrm{C}$ and annealed at each temperature for $2 \mathrm{~h}$ (samples 7 and 8, respectively), as shown in Figs. 1(a) and 1(d). The similar extinction of the polarization was also observed even in thinner films.

In order to understand the abnormal ferroelectric behavior of the thin film which underwent the melting and recrystallization, we have performed GIXD which provides us the information of both degree of crystallization and molecular orientation. Figure 2 shows a series of two-dimensional (2D) GIXD patterns of thin P(VDF-TrFE) films obtained after heat treatment with the routes illustrated in Fig. 1(a). The
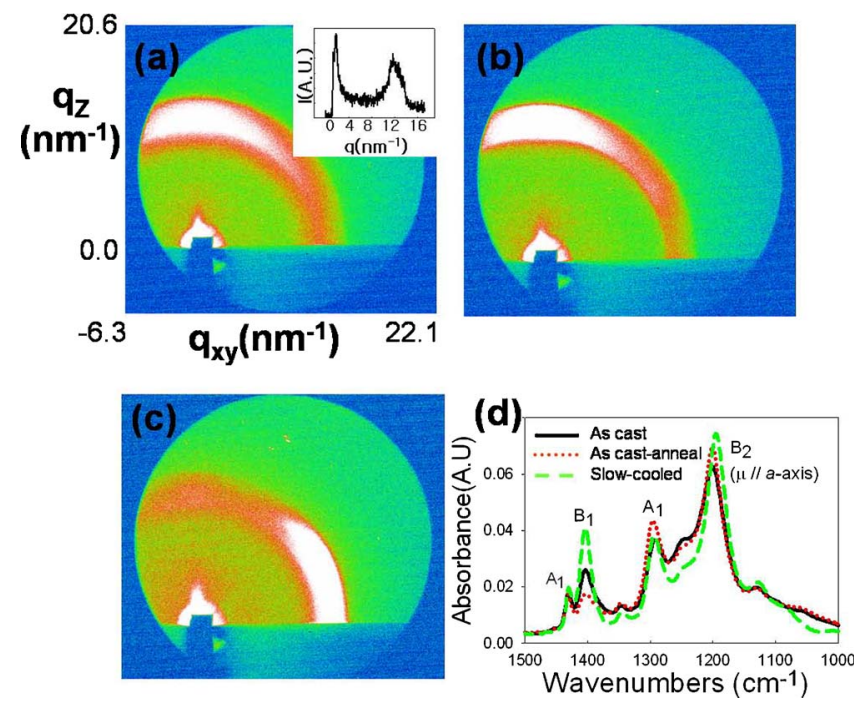

FIG. 2. (Color online) 2D GIXD patterns and GIRAS spectra for P(VDFTrFE) thin films with different annealing treatments: (a) as-cast films, (b) at $120{ }^{\circ} \mathrm{C}$, (c) at $170{ }^{\circ} \mathrm{C}$, and (d) GIRAS spectra. Inset of (a) displays an intensity profile along the vertical (meridian) direction.

patterns from the as-cast film [Fig. 2(a) and inset] and the annealed one at $120{ }^{\circ} \mathrm{C}$ [Fig. 2(b)] display the arced strong reflection near $q=12 \mathrm{~nm}^{-1}(q=4 \pi \sin \theta / \lambda)$ with twofold symmetry on the meridian which corresponds to both $\{110\}$ and $\{200\}$ reflections of orthorhombic P(VDF-TrFE) crystals. The orientation of the reflections arose from the preferential crystal orientation in which the $c$ axis, molecular chain axis, is preferentially aligned parallel to the substrate surface by the strong bias centrifugal force field generated during spin coating. In this situation, both $a$ and $b$ axes can rotate around the $c$ axis randomly. The external electric field applied in the polarization measurement induces the preferential orientation of the polar $b$ axis towards the electric field direction irreversibly, which is responsible for large permanent polarization of $\mathrm{P}(\mathrm{VDF}-\mathrm{TrFE})$.

The lower degree of crystallization of as-cast film than the annealed samples makes it difficult to induce the permanent polarization (see Table I). The crystallinity of the as-cast film is approximately two-thirds of that of the annealed samples. The GIXD patterns from the annealed samples all display the similar preferred orientation near meridian. Both improved crystallinity upon annealing and preferred orientation of the $c$ axis parallel to the substrate provide high remnant polarization, as shown in Figs. 1(b) and 1(c).

The sample heat treated above $T_{m}$ exhibits the different diffraction pattern from the ones observed previously. The preferred orientation of the $\{110\},\{200\}$ reflections is apparent near equator, indicating that the polymer crystals rotate $90^{\circ}$ around the incident beam. The altered orientation results in the preferred alignment of the molecular chain axis $c$ preferentially perpendicular to the substrate surface with both $a$ and $b$ axes parallel to the substrate. The rotation of crystals was observed in all the samples treated above $T_{m}$, irrespective of the cooling pathways (samples 6-8). The polar $b$ axis, parallel to the substrate in this situation, rarely responds to the external electric field, giving rise to nearly zero net polarization as shown in Fig. 1(d).

GIRAS provides spectroscopic evidence of the crystal orientation modification during heat treatment. Figure 2(d) shows GIRAS of approximately $75 \mathrm{~nm}$ thick samples pre- 
TABLE I. Ferroelectric properties and crystallinity for $\mathrm{P}(\mathrm{VDF}-\mathrm{TrFE})$ films annealed at various temperatures.

\begin{tabular}{|c|c|c|c|c|c|c|c|c|}
\hline $\begin{array}{l}\text { Temperature }\left({ }^{\circ} \mathrm{C}\right) \\
\text { /thickness }(\mathrm{nm})\end{array}$ & $25 / 250$ & $120 / 250$ & $130 / 250$ & $140 / 250$ & $150 / 250$ & $160 / 250$ & $135 / 110$ & $135 / 70$ \\
\hline \multicolumn{9}{|l|}{ Remnant polarization $^{\mathrm{a}}$} \\
\hline$\left[P_{r}\left(\mu \mathrm{C} / \mathrm{cm}^{2}\right)\right]$ & 1.2 & 7.75 & 8.35 & 8.73 & $0.55-1$ & $0.55-1$ & 11 & 8.33 \\
\hline Coercive voltage & & & & & & & & \\
\hline$\left[V_{c}(\mathrm{~V})\right]$ & N/A & 10.9 & 10.7 & 11.22 & N/A & N/A & 7.27 & 4.95 \\
\hline $\begin{array}{l}\text { Coercive field } \\
{\left[E_{c}(\mathrm{MV} / \mathrm{m})\right]}\end{array}$ & N/A & 43.56 & 42.96 & 44.88 & N/A & N/A & 66.09 & 70.71 \\
\hline $\begin{array}{c}\text { Crystallinity } \\
(\%)\end{array}$ & 45.89 & 59.48 & 63.22 & 63.77 & 60.5 & & & \\
\hline
\end{tabular}

${ }^{\mathrm{a}}$ The mean value from three different samples.

${ }^{\mathrm{b}}$ Calculated by the construction of crystallinity from the discrimination between the amorphous hump and the crystalline regions in GIXD curves.

pared with different conditions: one as cast, another annealed at $120^{\circ} \mathrm{C}$ for $3 \mathrm{~h}$, and the other slowly cooled from the melt. The annealing significantly increased the intensity of the band $\left(A_{1}, \boldsymbol{\mu} \| \mathbf{b}\right)$ at $1289 \mathrm{~cm}^{-1}$ whose vibrational transition moment is parallel to the $b$ axis. In addition, we observed the decrease of intensity at $1402 \mathrm{~cm}^{-1}\left(B_{1}, \boldsymbol{\mu} \| \mathbf{c}\right)$ band. These results indicate that the $c$ axis tends to orient parallel to the substrate surface. For the sample cooled slowly from the melt, the $c$ axis becomes perpendicular to the electrode surface confirmed by a significant intensity increase of $B_{1}$ band.

Electron microscopes permit direct visualization of the crystalline $\mathrm{P}(\mathrm{VDF}-\mathrm{TrFE})$ microstructure related to the molecular chain orientation. Edge-on crystalline microdomains closely packed with needlelike shape are obvious on the surface of the film spun coated and annealed at $130{ }^{\circ} \mathrm{C}$ for $2 \mathrm{~h}$ [Fig. 3(a)]. The average length and width of the crystals are approximately 300 and $50 \mathrm{~nm}$, respectively. The measurement of the light retardation using a polarized optical microscope (POM) suggests that the long axis of the crystals is parallel to the molecular chain axis ( $c$ axis). A needlelike crystal is composed of multiple stacks of crystalline lamellae along the long axis direction. GIXD, SEM, and POM results allow us to draw the schematic of the molecular and microstructural organization of the crystals, as shown in Fig. 3(c).

The microstructure of the film which underwent the melting and recrystallization is shown in Fig. 3(b). The surface was relatively smooth without a distinctive crystalline
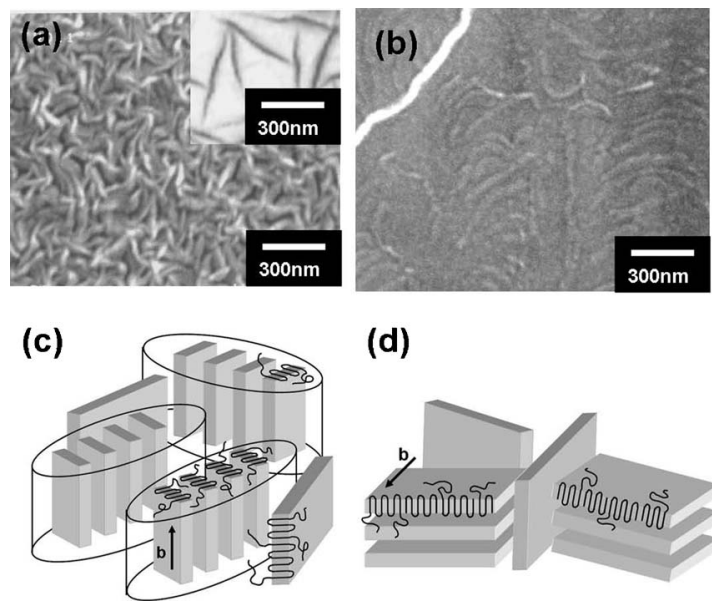

(d)

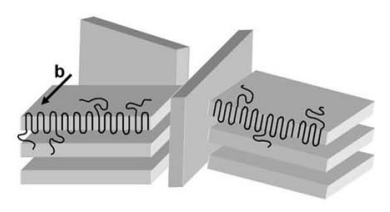

FIG. 3. (Color online) (a) SEM and TEM images (inset) of thin films annealed at $130{ }^{\circ} \mathrm{C}$ for $2 \mathrm{~h}$. (b) A microstructure image of the meltrecrystallized film. (c) A schematic of microstructure shown in (a). (d) Schematic of microstructure shown in (b). The polar $b$ axis is dominantly aligned parailei to the substrate. microstructure. The flat surface was attributed to the in-plane lamellar structure where the chain axis, $c$ axis, is dominantly normal to the substrate as confirmed by GIXD [Fig. 2(c)]. The polar $b$ axis aligned parallel to the substrate is ineffective for the electric field applied perpendicular to the substrate, leading to the null polarization [Fig. 3(d)]. The similar microstructural modification upon heat treatment occurred in thinner polymer films [the inset of Fig. 3(a)].

The molecular orientation of $\mathrm{P}(\mathrm{VDF}-\mathrm{TrFE})$ thin film with the polar $b$ axis of the crystals, which is responsible for ferroelectricity, preferentially normal to the electrode surface was completely transformed into one with the $b$ axis and the chain axis, $c$ axis, of the crystals parallel and perpendicular to the electrode surface, respectively, during melting and recrystallization, leading to the irreversible extinction of ferroelectric polarization. The modification of the crystal orientation which should be avoided for realizing a nonvolatile memory device of $\mathrm{P}(\mathrm{VDF}-\mathrm{TrFE})$ may be caused by the preferred interfacial energy between the crystalline $a b$ plane and the electrode.

This project was supported by the National Research Program for the 0.1 Terabit Non-volatile Memory Development sponsored by Korea Ministry of Commerce, Industry and Energy, Samsung Electronics, Co., Ltd. The x-ray experiments at PAL (4C2 beamline), Korea were supported by MOST and POSCO, Korea.

${ }^{1}$ O. Auciello, J. F. Scott, and R. Ramesh, Phys. Today 51(7), 22 (1998).

${ }^{2}$ T. J. Reece, S. Ducharme, A. V. Sorokin, and M. Poulsen, Appl. Phys. Lett. 82, 142 (2003).

${ }^{3}$ R. C. G. Naber, C. Tanase, P. W. M. Blom, G. H. Gelinck, A. W. Marsman, F. J. Touwslager, S. Setayesh, and D. M. de Leeuw, Nat. Mater. 4, 243 (2005).

${ }^{4}$ R. C. G. Naber, P. W. M. Blom, A. W. Marsman, and D. M. de Leeuw, Appl. Phys. Lett. 85, 2032 (2004).

${ }^{5}$ K. Noda, K. Ishida, A. Kubono, T. Horiuchi, H. Yamada, and K. Matsushige, J. Appl. Phys. 93, 2866 (2003).

${ }^{6}$ F. Xia, H. Xu, F. Fang, B. Razavi, Z.-Y. Cheng, Y. Lu, B. Xu, and Q. M. Zhang, J. Appl. Phys. 78, 1122 (2001).

${ }^{7}$ F. Xia, H. H. Xu, B. Razavi, Z.-Y. Cheng, and Q. M. Zhang, J. Appl. Phys. 92, 3111 (2002).

${ }^{8}$ K. Urayama, M. Tsuji, and D. Neher, Macromolecules 33, 8269 (2000).

${ }^{9}$ F. Xia and Q. M. Zhang, Appl. Phys. Lett. 85, 1719 (2004).

${ }^{10}$ Z.-M. Li, S.-Q. Li, and Z.-Y. Cheng, J. Appl. Phys. 97, 014102 (2005).

${ }^{11}$ Z. Li, Y. Wang, and Z.-Y. Cheng, Appl. Phys. Lett. 88, 062904 (2006).

${ }^{12}$ Q. M. Zhang, H. Xu, F. Fang, Z.-Y. Cheng, F. Xia, and H. You, J. Appl. Phys. 89, 2613 (2001).

${ }^{13}$ H. Xu, S. Ni, and C. Yang, J. Appl. Polym. Sci. 88, 1416 (2003).

${ }^{14}$ A. V. Bune, V. M. Fridkin, S. Durcharme, L. M. Blinov, S. P. Palto, A. V. Sorokin, S. G. Yudin, and A. Zlatkin, Nature (London) 391, 874 (1998).

${ }^{15}$ M. A. Barique and H. Ohigashi, Polymer 42, 4981 (2001). 